\title{
PROFESSOREN IN STAMMBÜCHERN - STAMMBÜCHER VON PROFESSOREN ROLLENKONSTELLATIONEN UND INSZENATORISCHE PRAXIS
}

\author{
WERNER WILHELM SCHNABEL
}

\section{PROFESSORS IN AUTOGRAPH BOOKS (ALBA AMICORUM) - AUTOGRAPH BOOKS OF PROFESSORS. CONSTELLATIONS OF ROLES AND STAGING PRACTICE}

\begin{abstract}
This study focuses on university professors as owners and inscribers in the autograph culture of the early modern period. It contains an analysis of autograph books (friendship books, alba amicorum) of professors as well as of professor inscriptions in student autograph books. It takes note of the form of the inscriptions, accompanying illustrations, and the role of these dedications in the communication practices within the academic setting. The study depicts autograph books as a distinctive form of social self-presentation closely bound to the academic and scholarly environment.
\end{abstract}

Keywords: university professors - students - autograph books (friendship books, alba amicorum) - communication self-presentation

DOI: $10.14712 / 23365730.2020 .21$

Alba Amicorum oder Stammbücher sind buchförmige Medien, die in den 1530er Jahren aufgekommen sind und unter wechselnden Bezeichnungen, in unterschiedlicher Form und bei variierender Trägerschaft bis in die Gegenwart existieren. Es handelt sich um überwiegend kleinformatige Alben mit leeren oder bordürengeschmückten Blättern, auf der Basis durchschossener Drucke oder auch aus leeren oder bedruckten Einzelblättern, die in buchähnlichen Kapseln, Schubern oder Kassetten aufbewahrt wurden. In ihnen sammelte der Besitzer handschriftliche, mitunter mit Illustrationen oder Notenzeilen versehene handschriftliche Notate von Personen, mit denen er bekannt geworden war oder gar Freundschaft geschlossen hatte. Ausgehend vom (zunächst nicht zwangsläufig akademischen) Umfeld der Wittenberger Reformatoren verbreitete sich die Stammbuchsitte rasch auch in anderen Milieus und Regionen. Früh schon wurde sie von der studierenden Jugend übernommen, fand bei Soldaten und beim Adel, bei Fürsten und Diplomaten Anklang, wurde von jungen Damen vornehmer Herkunft ebenso wie in humanistischen Kreisen aufgegriffen. Im Universitätsbereich fand sie für die nächsten knapp 300 Jahre ihre eigentliche Basis, ehe sich seit dem ausgehenden 18. Jahrhundert zunehmend auch bildungsfernere Milieus der Sitte bemächtigten und sie für die gebildeten Kreise und ihr Distinktionsbedürfnis unattraktiver machten. Durch die hohe Mobilität der Studenten verbreitete sich die Stammbuchpraxis seit 
den 1550er Jahren auch in Italien, Frankreich und den Niederlanden, erfasste Großbritannien, Dänemark, Schweden und die baltischen Länder ebenso wie Böhmen, Ungarn und den osteuropäischen Raum. Durch ihre Fundierung überwiegend in der gemeineuropäischen Verständigungssprache Latein übersprang sie die volkssprachlichen Grenzen ebenso wie konfessionellen Hürden, beschränkte sich lange allerdings auf die Universitätsstandorte und die großen Handelsstädte, die von Besuchern von weither aufgesucht wurden. Gleichwohl blieb die Albumsitte über lange Zeit ein Phänomen, das in erster Linie als ,deutsch' und als ,protestantisch' wahrgenommen und von Angehörigen anderer Kulturen oft etwas skeptisch beurteilt wurde - was nicht zuletzt in der Form der Einträge zum Ausdruck kommt, die den strikten Konventionen des regelgerechten Albumeintrags dann oft nicht entsprachen.

Stammbücher, die oft auf Reisen mitgeführt und an verschiedenen Aufenthaltsorten genutzt wurden, präsentieren sich vordergründig als Werkzeuge der Memoria und sind das ist in der internationalen Forschung seit langem bekannt - tatsächlich ein wichtiger Faktor privater und milieuspezifischer Erinnerungskultur. Vom zweiten Drittel des 16. bis ins 21. Jahrhundert sind die Alben gängige Mittel gewesen, Erinnerungen an zwischenmenschliche Begegnungen zu konservieren und später bei Bedarf abrufen zu können. Ein solcher Interpretationsansatz, der zumindest seit dem frühen 19. Jahrhundert weitgehend dominant geworden ist, bezieht sich in erster Linie auf die Perspektive des Stammbuchhalters. Schließlich ist es ja dieser, der die einschlägigen Einträge akquiriert, sammelt und später zur Rückerinnerung nutzt.

Dass eine solch simple Einordnung mehr als problematisch ist, zeigt sich allerdings dann, wenn man die Stammbuchpraxis als ein Kommunikationsphänomen in Augenschein nimmt. Das erfordert den Blick nicht nur auf eine der beteiligten Instanzen, sondern die Einbeziehung aller Beteiligten. Neben dem Sammler, der als primärer Adressat eines Textes fungiert, ist dies zumindest der Inskribent, der um einen Beitrag gebeten worden ist, in aller Regel aber auch ein Publikum, das in die Kommunikation einbezogen wird. Zudem erfolgt die Informationsvermittlung nicht etwa mündlich, also auf Synchronizität angelegt; vielmehr impliziert sie eine asynchrone Wahrnehmung und ist auf Dauerhaftigkeit ausgelegt, hat also unmittelbare und zeitgenössische ebenso wie zukünftige Rezeptionen zu berücksichtigen. Mindestens ebenso sehr wie dem Sammelmedium sollte deshalb zunächst einmal dem einzelnen Eintrag besonderes Interesse gelten. Immerhin ist er der Träger der Botschaft, die - rein textuell oder mit Bild- oder Notenbeigaben versehen - vom Sender an den bzw. die Empfänger vermittelt wird. Alle beteiligten Instanzen können dabei eigenständige, durchaus unterschiedliche Perspektiven und zum Teil auch verdeckte Intentionen haben. ${ }^{1}$ Die aufgrund einer langdauernden Wahrnehmungsgeschichte naheliegende Festlegung des Stammbuchs auf die Funktion als Erinnerungsanreiz, wie sie vom Albumhalter (und nicht selten auch von den Inskribenten) in ritualisierter Weise betont wird, ist deshalb

1 Zum hier verwendeten methodischen Ansatz ausführlich Werner Wilhelm SchnaBel, Das Stammbuch. Konstitution und Geschichte einer textsortenbezogenen Sammelform bis ins erste Drittel des 18. Jahrhunderts, Tübingen 2003 (Frühe Neuzeit, 78). Grundlegend auch Marie Ryantová, Památníky aneb štambuchy, to jest alba amicorum. Kulturně historický fenomén raného novověku [Stammbücher, d.h. alba amicorum. Kulturhistorisches Phänomen der Frühen Neuzeit], České Budějovice 2007 (Monographia historica, 8), sowie Marie Ryantová, Frühneuzeitliche Stammbücher als Mittel individueller Selbstdarstellung, Frühneuzeit-Info 18, 2007, S. 91-108. 
zwar nicht falsch, aber doch zu grob, um die komplexe Stammbuchpraxis angemessen bewerten zu können.

Tatsächlich haben die beiden unmittelbar beteiligten Akteure in der Eintragssituation Interessen, die sich zumindest zum Teil sehr wohl unterscheiden und in der Niederschrift idealerweise einen Ausgleich finden müssen. Der Albumbesitzer, der sein Stammbuch mit der Bitte um einen Eintrag vorlegt, möchte gerne ein schön geschriebenes und aussagekräftiges Notat erhalten. Unter Gleichrangigen ist es wesentlich, dass der Einträger nach Wertvorstellungen, Rollenverhalten und sozialem Status in den Kreis der bereits versammelten Bekannten passt; erwartet wird zudem in der Regel die Dokumentation einer engeren Verbindung zwischen Schreiber und Empfänger, für die das lateinische Wort ,amicus', aber auch das deutsche ,Freund' allerdings nur schwache Signale sind - beide werden in der Frühneuzeit noch nicht in dem emphatischen Sinne gebraucht wie seit dem späteren 18. Jahrhundert und markieren im allgemeinen eher eine Zweckgemeinschaft. ${ }^{2}$ Die zumindest bis in ebendiese Zeit mit Vorliebe akquirierten Beiträge höherrangiger Schreiber hingegen spekulieren vor allem auch auf die Prominenz der Personen, mit deren ,Freundschaft' oder Patronage man sich später rühmen kann. Gerade in diesen Fällen wird erwartet, dass der Schreiber möglichst auch einige Lobesworte hinterlässt, die seine Wertschätzung des Albumhalters zum Ausdruck bringen. Inhaltlich erwünscht sind in diesem Fall nicht nur pointierte, sondern auch ernsthafte, nach gängigem Wertsystem unangreifbare Maximen, die etwas von der Eigenart des Schreibers transportieren; gerne gesehen sind statushebende Beigaben, die meist nicht nur Mühe, sondern auch Kosten verursachen: eine von einem spezialisierten Künstler eingemalte Wappenminiatur etwa oder eine allegorische Darstellung. Schließlich wird die Inskription nicht nur vom Adressaten selbst eingesehen, sondern von einer Halböffentlichkeit zur Kenntnis genommen. Diese schließt von der Art der Einträge, dem an den Tag gelegten Aufwand und dem Kreis der versammelten Personen nicht zuletzt auf Eigenschaften und Geltung des Stammbuchhalters selbst zurück. ${ }^{3}$

Der Inskribent auf der anderen Seite ist im Regelfall nicht etwa nur ein Schreiber, der pflichtschuldig einen Kurztext spezifischer Form abliefert. Vielmehr ist er zunächst einmal Teil der angedeuteten Halböffentlichkeit. Er betrachtet die bereits vorhandenen Inskriptionen mit Neugier oder Interesse, sucht sich nach meist sozialhierarchischen, gegebenenfalls aber auch herkunftstopographischen oder netzwerktechnischen Gesichtspunkten seinen Platz innerhalb der vorgefundenen Strukturen und wählt seinen Eintragstext durchaus bewusst; gelegentlich nimmt er in seinem Text sogar inhaltlich auf Nebeneinträge Bezug. ${ }^{4}$

2 Immer noch grundlegend Wolfdietrich RASCH, Freundschaftskult und Freundschaftsdichtung im deutschen Schrifttum des 18. Jahrhunderts. Vom Ausgang des Barock bis zu Klopstock, Halle/S. 1936 (Deutsche Vierteljahrsschrift für Literaturwissenschaft und Geistesgeschichte, Buchreihe, 21). Weiter vgl. Wolfgang ADAM, Wieder gelesen: Wolfdietrich Rasch: Freundschaftskult und Freundschaftsdichtung im deutschen Schriftum des 18. Jahrhunderts, in: Ferdinand van Ingen - Christian Juranek (Hgg.), Ars et Amicitia. Beiträge zum Thema Freundschaft in Geschichte, Kunst und Literatur. Festschrift für Martin Bircher zum 60. Geburtstag am 3. Juni 1998, Amsterdam - Atlanta 1998 (Chloe, 28), S. 41-55.

3 Vgl. Werner Wilhelm Schnabel, Stammbuch-Schelte. Theodor Lebrecht Pitschel und seine „, Gedanken über die Stammbücher“, in: Klára Berzeviczy - Péter Lőkős - Zsófia Hornyák (Hgg.), „Ars longa, vita academica brevis“. Studien zur Stammbuchpraxis des 16.-18. Jahrhunderts, Budapest 2009 (Vernetztes Europa, 6), S. $47-73$.

4 Exemplarisch Gilbert Hess, Literatur im Lebenszusammenhang. Text- und Bedeutungskonstituierung im Stammbuch Herzog Augusts des Jüngeren von Braunschweig-Lüneburg (1579-1666), Frankfurt/M. u.a. 2002 (Mikrokosmos, 67). 
Dabei wägt er sehr wohl ab, wie er sich in dem gereichten Album dauerhaft verewigen möchte. Denn der Text und der Eindruck, den er mit seinem Eintrag von sich erweckt, wird über Jahre, Jahrzehnte oder gar Jahrhunderte fixiert sein. Der Schreiber hat also ein dringendes Interesse daran, nicht nur den Wünschen seines direkten Gegenübers entgegenzukommen, sondern auch die spätere Rückerinnerung an sich zu beeinflussen bzw. ein bestimmtes Bild von sich zu installieren. In der Wahl seines Themas, der eingetragenen Sentenz, der Heranziehung autoritativer Referenzen, dem Gebrauch bestimmter Sprachen, in der ,Innigkeit' seiner Dedikationspassage und der Ausführlichkeit seiner Unterschrift samt Herkunfts- und Amtsbezeichnungen beeinflusst er die spätere Wahrnehmung seiner selbst. Und die beschränkt sich wiederum nicht auf den unmittelbaren Adressaten, sondern umfasst eine engere oder weitere Gruppe von Beteiligten, die von den Einträgen jetzt oder in Zukunft Kenntnis nehmen werden. Auch deren Wertorientierungen, Denkweisen, Reaktionen und Einschätzungen gilt es zu berücksichtigen. Dass Stammbucheinträge über lange Zeit rezipiert werden und die spätere Rückerinnerung bestimmen werden, ist ein Topos, der in zahlreichen Dedikationspassagen ausdrücklich thematisiert wird; dass sie zugleich die Wahrnehmung und Einschätzung des Schreibers beeinflussen, ja steuern sollen, wird freilich oft eher verdeckt deutlich.

So wie der Albumhalter also bestimmte Interessen verfolgt, wenn er sein Stammbuch vorlegt, so gilt dies auch für den Inskribenten, der sich darin verewigen soll. Der Stammbucheintrag ist eine Sprachhandlung, die mit den Mitteln der Belehrung und der Herstellung von Nähe oder Distanz nicht zuletzt der Selbstdarstellung und Positionierung, der Erinnerungssetzung und Erinnerungssteuerung dient. Er ist alles andere als intim und im emphatischen Sinne persönlich; immer hat sein Verfasser auch die aktuell oder künftig beteiligten Einträger und Betrachter zu kalkulieren, die sich anhand der niedergeschriebenen Zeilen ein Urteil über die Beteiligten bilden.

Ähnliches gilt für die Leserschaft der Einträge, die nur zum Teil mit den Inskribenten identisch ist. Bekannt ist etwa der Umstand, dass Stammbücher mit großem Interesse als Sammlungen lehrreicher Sentenzen gelesen wurden, also durchaus eine Art Lebenshilfefunktion zugeschrieben bekamen. ${ }^{5}$ Notate, die diesem hochgesteckten Ideal weniger oder gar nicht entsprachen, kamen so leicht in Misskredit und provozierten wenig schmeichelhafte Rückschlüsse auf Schreiber und Albumhalter. ${ }^{6}$ Wenn die einen gerade auch nach prominenten, ja berühmten Inskribenten suchten und gewissermaßen die ,Aura' einer handschriftlichen Äußerung goutieren wollten, gerieten die Alben dadurch bei anderen in den Ruf, einer bloßen Autographensammelwut zu dienen; insbesondere im 19. Jahrhundert führte dies immer wieder zu ironischen oder kritischen Einträgen von Berühmtheiten, die es für nötig hielten, damit ihre Distanz zur Sammelpraxis zu markieren. Über weite Teile der Stammbuchgeschichte wurde den Alben also keineswegs die Erwartung von

5 Vgl. Herbert Kater, Das Stammbuch August von Goethes, Einst und Jetzt 35, 1990, S. 251. In diese Richtung zielt auch das Analysekonzept einer , additiven Morallehre': Wolfgang Harms, Rezension zu Hans Bots - Giel van Gemert (Hgg.), L'Album Amicorum de Cornelis De Glarges 1599-1683 [...], Amsterdam 1975, Daphnis 6 , 1977, S. 400-402, hier S. 402.

$6 \mathrm{Zu}$ Lesererwartungen und deren Folgen für die Einschätzung des Mediums W. W. Schnabel, Stammbuch-Schelte, S. 47-73. 
,Privatheit' oder ,Intimität' entgegengebracht, die man in jüngeren Zeiten gerne hineininterpretiert hat. ${ }^{7}$

Diese Sachverhalte gilt es im Auge zu behalten, wenn im Folgenden von Einträgen des universitären Lehrpersonals in studentischen Alben die Rede ist, wie sie zu vielen Tausenden erbeten und gesammelt worden sind. ${ }^{8}$ Die Spielräume, die sich hierbei ergeben, lassen sich auf beschränktem Raum immerhin exemplarisch zeigen. ${ }^{9}$

$$
* * *
$$

Auffällig ist es, dass viele Professoreneinträge des späteren 16. und frühen 17. Jahrhunderts vergleichsweise ausführlich sind. Bei den frühesten Inskriptionen, die seit den 1530er Jahren an der Universität Wittenberg gesammelt wurden, nehmen die Notate sogar oft großformatige Buchseiten ein, da häufig gedruckte Bücher - etwa die Bibelübersetzung Luthers oder Melanchthons ,Loci communes' - dafür herangezogen wurden. Die Schreiber, deren Handschriften übrigens zunächst durchaus nicht von Studenten, sondern von bürgerlichen Bittstellern gesammelt wurden, fungierten hier weniger als Professoren denn als Seelsorger und genossen aufgrund ihres Status als Reformatoren besondere Verehrung. Infolgedessen wandten Luther, Melanchthon und ihr akademisches Umfeld große Mühe darauf, jedem Bittsteller individuelle Beiträge zu verfassen. Diese nahmen offensichtlich auf die jeweilige Situation des Empfängers Bezug. Mit ihrer exegetischen Struktur aus (Bibel-) Zitat, lehrhafter Auslegung, (nicht immer formulierter) Zueignung und Namensnennung des Schreibers begründeten sie einen besonderen Eintragstypus, der für Reformatoreneinträge charakteristisch blieb. ${ }^{10}$ Schüler und Weggefährten der prominenten Theologen wie Johann Aurifaber (1519-1575) oder Georg Rörer (1492-1557) haben derlei Texte schon zeitgenössisch im Druck zugänglich gemacht und dabei deren lehrhafte und seelsorgerliche Bedeutung unterstrichen, die damals noch neue Praxis des handschriftlichen Eintrags in dargereichte Bücher aber nicht eingehender thematisiert. ${ }^{11}$ Erst in den 1560er Jahren - mit

7 Vgl. W. W. Schnabel, Das Stammbuch, S. 157.

8 Basis für die Recherche nach Stammbüchern ist heute üblicherweise das Repertorium Alborum Amicorum (RAA), die weltweit größte Online-Datenbank für Stammbücher und Stammbuchfragmente in öffentlichem und privatem Besitz (<https://raa.gf-franken.de/de/>). Der Datenbestand dokumentiert in unterschiedlicher Dichte (Stand August 2019) über 25.500 historische Medieneinheiten mit Standortangaben und Hinweisen auf die jeweils dazu erschienene Literatur. Außerdem sind derzeit rund 267.500 Albumeinträge mehr oder minder ausführlich nachgewiesen. Neben diversen Zusatzinformationen (Projektbeschreibung, umfangreiches Literaturverzeichnis zum Thema, Dokumentation von Ausstellungen seit 1882) bietet das Portal seit Anfang 2019 auch die Möglichkeit, Albumdigitalisate zu hosten - ein kostenfreies Angebot, das sich v.a. an kleinere Institutionen, aber auch an Sammler richtet, die sonst nur schwerlich die Möglichkeit haben, derlei Dokumente öffentlich bekannt zu machen. - Eine Recherche nach dem Suchbegriff „Prof[.]“ ergab im Februar 2019 über 5800 Treffer; viele weitere kommen hinzu, wenn man die gängige Abkürzung „P. P. [O.]“ hinzunimmt. Zu berücksichtigen ist allerdings, dass sich Professoren nicht notwendigerweise auch als solche zu erkennen gegeben haben müssen (siehe unten).

9 Zum Quellenwert speziell akademischer Alba amicorum vgl. Werner Wilhelm SchnaBeL, Stammbücher, in: Ulrich Rasche (Hg.), Quellen zur frühneuzeitlichen Universitätsgeschichte. Typen, Bestände, Forschungsperspektiven, Wiesbaden 2011 (Wolfenbütteler Forschungen, 128), S. 421-452.

10 W. W. Schnabel, Das Stammbuch, S. 260-265.

11 Johann Aurifaber, Auslegung/ etzlicher Trostsprüche/ so der Ehrwirdige Herr/ Doctor Martinus Luther/ inn seiner lieben Herrn/vnd guten Freunde Bibeln vnd Postillen/mit eigener handt (zu seinem gedechtnis) geschrieben, [Erfurt] 1547; [Georg RöRER (Hg.).] Vieler schöner Sprüche aus Göttlicher Schrifft auslegung/ daraus Lere vnd Trost zu nemen/Welche der ehnrwirdige [!] Herr Doctor Martinus Luther seliger/vilen in jre Biblien geschrieben. Dergleichen Sprüche von andern Herrn ausgelegt/sind auch mit eingemenget, Wittenberg 1547. 
dem Absterben der Reformatorengeneration - verschwand dieser Eintragstypus wieder. Nun setzte sich im akademischen Bereich das über Jahrhunderte konstant bleibende Strukturmuster von Sentenz/Gedicht und Dedikation durch, das trotz seines vorgegebenen Bauschemas große Freiheiten zur Ausgestaltung, Akzentuierung und Ergänzung durch weitere textuelle, bildliche oder notenschriftliche Elemente ließ.

In den (professoralen) Reformatoreneinträgen wie in den späteren Inskriptionen des universitären Lehrpersonals signalisierte schon der schreiberische ,Aufwand' eine deutliche Zugewandtheit zum Empfänger, obwohl dieser sozial ja in aller Regel deutlich unter dem Inskribenten stand. Im Falle eines Eintrags des Altdorfer Orientalisten Daniel Schwenter (1585-1636) von 1623 war der Empfänger nicht einmal ein Angehöriger der Universität, sondern ein Lauinger Maler, Zeichner und Astronom, der sich aus Glaubensgründen eben zur Übersiedelung in die Reichsstadt Nördlingen entschlossen hatte. ${ }^{12}$ (Abb.1) Zur Herstellung von ,Nähe' gehörte es zum Beispiel, dass die Inskribenten eine vergleichsweise umfangreiche Dedikationsformel einsetzten, in der sie den Adressaten in aller Regel namentlich apostrophierten und mit freundlichen Epitheta ornantia versahen - gelobt fanden sich beispielsweise die Begabung oder der Fleiß des Albumhalters, in Fällen prominenter Herkunft auch der Umstand, dass der Sohn ein würdiger Nachfahre seines Vaters sei, der einst selbst eine große Zukunft vor sich habe. ${ }^{13}$ Zugleich legten die Schreiber aber auch Wert darauf, ihren eigenen Rang ausführlich zu dokumentieren. Der Status als Professor, die Nennung der Denomination und gegebenenfalls noch weiterer akademischer oder höfischer Ehrenämter - nicht selten des Rektorats, das eben ausgeübt wurde - gehörten zu den Standardbestandteilen der Dedikationen. Sie rubrizierten die Niederschrift oft ausdrücklich (und in Übereinstimmung mit dem sozialen Komment) als Zeichen freundlicher Zugewandtheit und Achtung. Im gezeigten Beispiel kam hinzu, dass statt einer expliziten Datierung ein - immerhin aufwendig ersonnenes - Chronogramm angefügt wurde, das auf die Situation des Empfängers als Exulant Bezug nahm und auf Gott als Schutzinstanz des Lebensweges verwies. Der Eintrag scheint also tatsächlich speziell für den Empfänger entworfen und formuliert worden zu sein, der sich so einer direkten Ansprache durch den Hochschullehrer rühmen durfte.

Mindestens ebenso auffällig ist im übrigen die Technik, schon im Hauptteil des Eintrags, der üblicherweise aus einer Sentenz oder einem kurzen Gedicht bestand, weise Einsichten in den Lauf der Welt, Moralregeln oder Handlungsanleitungen unterzubringen, die den Habitus der Ernsthaftigkeit und Gesetztheit des Schreibers unterstreichen sollten. Anders als studentische oder soldatische Einträger, die nicht selten auch einmal weniger angepasste Äußerungen hinterließen, stellten sich die Professoren standesgemäß als fromme, überlegene und zugleich weltkluge Persönlichkeiten dar, die es geradezu als ihre Aufgabe ansahen, dem jüngeren Empfänger Ratschläge und Erfahrungstatsachen ans Herz zu legen. Sehr häufig nehmen diese Sentenzen und Merksätze erwartungsgemäß auf die eigene Disziplin

12 Zum Albumhalter Georg Brentel (1581-1634) siehe Allgemeines Künstlerlexikon 14, München u.a. 1996, S. 134. Sein Stammbuch in Wien, Museum für Angewandte Kunst, Bibliothek: Q I 7.

13 Vgl. derartige Zuschreibungsmöglichkeiten bei Werner Wilhelm ScHNABEL, Heteronomie und Surrogatcharakter des Kinderstammbuchs. Gustav von Racknitz (1635-1681) und sein Album, Daphnis 19, 1990, S. 423-470; Ders., Selbstinszenierung in Bildern und Texten. Stammbücher und Stammbucheinträge aus Helmstedt, in: Jens Bruning - Ulrike Gleixner (Hgg.), Das Athen der Welfen. Die Reformuniversität Helmstedt 1576-1810, Wolfenbüttel 2010, S. 68-77, hier S. 69-72. 
Bezug, dokumentieren in Inhalt, Metaphorik oder zitierter Autorität also das Fachgebiet des Schreibers, in dem er sich besonders gut auskannte - hier etwa mit einem aufmunternden Zitat des Kirchenvaters Ambrosius von Mailand. ${ }^{14}$ Freilich beschränken sich die Texte keineswegs auf eine solch wohlwollende Moralisatio. Denn zugleich arbeiten sie nicht selten mit einer dezidierten Beeindruckungsstrategie. Wenn Schwenter in seinem Eintrag an den Lauinger Maler neben dem Lateinischen, das Brentel bestens verstand, auch verschiedene arabische Sprachen bzw. Schriftsysteme einsetzte, so konnte er sicher sein, dass sein Gegenüber diese Passagen in keiner Weise entziffern, verstehen oder nachvollziehen konnte. Sie dienten also eher als demonstrativer Ausweis eigenen Wissens und Könnens gegenüber anderen Lesern, nicht aber als direkte Ansprache an den Empfänger, der die fremdartigen Zeichen allenfalls mit Bewunderung für den gelehrten Einträger zur Kenntnis nehmen konnte. Ähnliche Überwältigungsverfahren lassen sich immer wieder etwa mit der Wahl sehr entlegener, alles andere als kanonischer Gewährsleute feststellen und dienten wohl vor allem dazu, den Wissensvorsprung des Schreibers gegenüber dem Leser sichtbar zu machen.

Professoreneinträge des späteren 17. und 18. Jahrhunderts haben diese Verfahrensweisen im Prinzip durchaus beibehalten, auch wenn sie sich im Hinblick auf den Schreibaufwand zunehmend zurückgenommen haben. Vergleichsweise freundlich und mit der Formulierung guter Wünsche auch noch sehr zugewandt ist etwa das Notat des Altdorfer Theologieprofessors Johann Andreas Sixt im Jahr 1777. Er amtierte damals als Rektor - ein häufiger Anlass, bei dem auch fachfremde Studenten - hier der 20jährige Medizinstudent Johann Friedrich Seiferheld $(* 1757)^{15}$ - um einen Eintrag baten. (Abb.2) In einer Zeit, in der das Deutsche auch im akademischen Unterricht schon dominant geworden war, hielt er immer noch an der lateinischen Gelehrtensprache fest, ja er zitierte den neutestamentlichen 2. Paulusbrief an Timotheus sogar in seiner griechisch-hellenistischen Urfassung - was einen historisch arbeitenden Philologen natürlich ziert. Minder traditionalistische oder auf die Dokumentation fachlicher Expertise ausgerichtete Professoren wie der Altdorfer Jurist Johann Albrecht Spieß (1704-1766) nahmen dagegen schon in den 1730er Jahren keinen Anstand, auch ihre Stammbucheinträge auf Deutsch zu verfassen. ${ }^{16}$ (Abb.3) Im akademischen Milieu blieben sie damit allerdings bis auf weiteres in der Minderheit. Dort hielt man sich bis ins ausgehende 18. Jahrhundert und zum Teil auch darüber hinaus an die lateinische Gelehrtensprache, die dem eigenen Status am ehesten gerecht zu werden schien.

Natürlich sind distanzierte Professoreneinträge auch schon in früherer Zeit vorgekommen. ${ }^{17}$ Gut dokumentiert ist das Beispiel des niederländischen Latinisten und Gräzisten Daniel Heinsius (1580-1655), der sich in späteren Lebensjahren - offenbar bei zunehmender beruflicher Belastung - meist auf eine kurze Sentenz beschränkte (,, Quantum est, quod nescimus"), die er immer wieder eintrug und auf diese Weise geradezu in der Art eines

14 „Nescit tarda molimina Sancti Spiritus gratia“ (Ambrosius, Expositio in Lucam II, 19 = Migne, Patrologia Latina 15,1560$)$.

15 Seiferheld war später Stadtphysikus in seiner Geburtsstadt Schwäbisch Hall. Sein Stammbuch in Straßburg, Bibliothèque Nationale et Universitaire: Ms. 2.143.

16 Empfänger war der Student und spätere Buchhändler bzw. Verleger in Altdorf Lorenz Schüpfel (1716-1789). Sein Stammbuch in Erlangen, Universitätsbibliothek: Ms. 1371.

17 Beispielsweise (wie viele andere Einträger in diesem Album) Dethard Horst (1546-1618) bei David Ulrich (1561-1626), fol, 20r; siehe Wolfgang Klose u.a., Wittenberger Gelehrtenstammbuch. Das Stammbuch von Abraham und David Ulrich. Benutzt von 1549-1577 sowie 1580-1623, Halle 1999, hier S. 65 f. 
Symbolums verwendete. ${ }^{18}$ Diese pragmatische Arbeitserleichterung ist umso auffälliger, als er gegenüber seinen humanistischen Freunden zumindest bis um 1605/1610 durchaus den Ehrgeiz an den Tag legte, mit kunstvollen, speziell für diese Situation geschaffenen lateinischen Gedichten und ausgewählten griechischen Literaturzitaten zu brillieren. Die Diskrepanz zwischen der poetischen Kunstübung für ein seelenverwandtes Publikum, das derlei Poeme durchaus als literarische Artefakte wahrnahm, und den extemporierten Kurzeinträgen für die akademische ,Laufkundschaft' ist durchaus auffällig. Auch Heinsius, der seinerseits ambitionierte niederländische Verse verfasste, behielt in den Albumeinträgen übrigens durchgängig die lateinische und griechische Sprache bei, positionierte sich in diesem Medium also als akademischer Philologe traditioneller Prägung, nicht als ,moderner' und volkssprachlicher Poet.

Tatsächlich wurden die deutlich distanzierteren und zum Teil wohl geradezu fließbandartig erzeugten Inskriptionen im 18. Jahrhundert immer gängiger. ${ }^{19}$ Sie zeigen ganz deutlich, dass der soziale und mentale Abstand zwischen Lehrpersonal und Studierenden größer wurde und ein direktes Lehrer-Schüler-Verhältnis gerade an den größeren Universitäten offenbar zunehmend den Bedingungen anonymerer Bildungsinstitutionen wich. Charakteristisch ist etwa die starke Reduzierung der Zueignungspassage auf eine kurze, kaum noch aussagekräftige Formel, die auf die Benennung des Adressaten und lobende Epitheta verzichtete, ja die Abkürzung des eigenen Vornamens und das Weglassen eigener Statusangabe. Auf diese Weise trug sich 1747 zum Beispiel der Mathematiker Leonhard Euler (1707-1783) im Album des reisenden Studenten und späteren Enzyklopädisten Johann Georg Krünitz (1728-1796) ein. ${ }^{20}$ (Abb.4) Auf solche Weise wurden augenscheinlich Besucher abgefertigt, zu denen die Schreiber keinen direkteren Kontakt mehr unterhielten und die sie in erster Linie als lästige Autographensammler wahrnehmen mochten. Der Freude des Empfängers über die handschriftlichen Zeilen des prominenten Professors tat dies allerdings keinen Abbruch - er ergänzte nicht nur selbst den Vornamen des schreibfaulen Inskribenten, sondern vermerkte akribisch auch dessen Geburtsort und -datum und schließlich seinen erst 36 Jahre später erfolgten Tod. Ein derartiges Interesse an den späteren Schicksalen der Einträger, zu denen man nicht unbedingt ein engeres Verhältnis unterhalten haben musste, war keineswegs nur die Eigenart eines besessenen Materialsammlers wie Krünitz, sondern durchaus verbreitet. Es belegt die Weiternutzung der Alben als Lese- und Erinnerungsmedien über Jahrzehnte hinweg, also auch in Zeiten, in denen keine neuen Einträge mehr akquiriert wurden. ${ }^{21}$

Selbst gegenüber adeligen Studenten, denen gegenüber traditionell ja sehr viel verbindlicher aufgetreten wurde, nahm man eine solche Distanzhaltung ein. Das zeigt das Beispiel

18 Barbara Becker-Cantarino, Die Stammbucheintragungen des Daniel Heinsius, in: Jörg-Ulrich Fechner (Hg.), Stammbücher als kulturhistorische Quellen, München 1981 (Wolfenbütteler Forschungen, 11), S. 137-164. Die dort auf S. 161-163 chronologisch aufgelisteten Belege lassen sich mittlerweile leicht ergänzen.

19 Weitere einschlägige Beispiele aus den 1730er Jahren - z. T. mit vollständigem Verzicht auf eine Dedikationspassage - bei Werner Wilhelm SchnaBeL, Johann Wilhelm Bergius (1713-1765) und die Berliner Frühaufklärung. Personale Konstellationen in Zeiten kulturellen Horizontwandels, Jahrbuch für brandenburgische Landesgeschichte 67, 2016, S. 91-142, hier Nr. 16, 18, 19, 21.

20 Sein Stammbuch in London, The British Library: Add. Ms. 18.713. Eingehend zu seinen beiden Alben Carl Joachim Classen (Hg.), Die Stammbücher von Johann Georg Krünitz (1728-1796), hg. von Roswitha Classen, Stuttgart 2017.

21 Zu den ,Gebrauchsphasen und -situationen' W. W. Schnabel, Das Stammbuch, S. 160-165. 
des berühmten Altphilologen Christian Gottlieb Heyne (1729-1812) im Album des aus Ungarn stammenden Juristen Sándor (Alexander) von Podmaniczki. ${ }^{22}$ Der Schreiber legte hierbei nicht nur eine recht flüchtige Handschrift an den Tag, sondern kürzte seine Zueignungsformel, in der er immerhin Erinnerung und Ehrung als Beweggründe namhaft machte, auch auf größtmögliche Weise zusammen; dass auch er auf eine volle Namensnennung und die im sonstigen Verkehr üblichen Statusangaben verzichtete, ist vielsagend. Eine ähnliche Praxis lässt sich bei vielen weiteren Einträgen in diesem zweibändigen Stammbuch feststellen, das der ungarische Baron auf seinen ausgedehnten Reisen durch das Reich mitführte und füllen ließ. Tatsächlich beschränkte er sich dabei auf Professoreneinträge, verzichtete also - ungewöhnlicherweise - auf die Notate von Kommilitonen, mit denen er zwangsläufig ja auch Bekanntschaft schloss. (Abb.5) Der Anteil solch distanzierter Inskriptionen seitens der Universitätsprofessoren wächst im Laufe des 18. Jahrhunderts zusehends und auffälligerweise. Vielleicht ist das auch einer der Gründe dafür, dass professorale Einträge mehr und mehr aus den studentischen Alben verdrängt wurden. Dazu trug sicher auch eine Auseinanderentwicklung der milieuspezifischen Wertsysteme bei, die in der jungen Generation zunehmend von anderen, nicht zuletzt literarisch unterfütterten Habituskonzepten bestimmt wurden. Inhalte und ,Ton' der studentischen Einträge entwickelten sich in eine Richtung, die mit der traditionellen moralisierenden Ausrichtung akademischer Alben nur noch teilweise in Übereinstimmung zu bringen waren. ${ }^{23}$ Der anakreontische Lobpreis von Wein, Weib und Gesang, die offene Propagierung epikuräischen Lebensgenusses und erotische Zweideutigkeiten, tränenreiche Freundschaftsbeschwörungen oder die Zurschaustellung dissozialer, forciert ,burschikoser' Verhaltensweisen wurden beliebt - schränkten aber natürlich auch die Möglichkeit ein, derartige Verlautbarungen seinen akademischen Lehrern zur Kenntnis zu bringen. So führten die Stammbuchhalter nicht selten zwei Alben parallel, die den unterschiedlichen Bezugsgruppen vorbehalten waren. Mehr und mehr beschränkten sie sich dann aber auf handschriftliche Notate ihrer Studienkollegen. Deren Inhalte durften eine wesentlich größere Spannweite aufweisen und waren oft nicht mehr mit den Maßstäben traditioneller Verhaltensmaximen zu messen.

Allerdings können solche Äußerungen ebenso wenig wie die der Professoren als unmittelbare Widerspiegelung einer individuellen Persönlichkeit missverstanden werden. ,Authentizität' oder Wahrhaftigkeit kann weder im einen noch im anderen Fall ohne weiteres unterstellt werden. Die vermeintlich unangepassten Studenten spielten ebenso wie die Professoren eher mit bestimmten Habituskonzepten, die man sich schuldig zu sein glaubte, mit denen man sich einer Gruppe zuordnen und dem späteren Leser im Gedächtnis bleiben wollte. Es handelte es sich um einen gruppenspezifischen Komment, um das Einnehmen von Rollen, die dem jeweiligen Stand und Alter und der aktuellen Position im gesellschaftlichen Gefüge angemessen zu sein schienen bzw. mit denen man literarisch vermittelte Verhaltensweisen und eskapistische Phantasien spielerisch nachvollzog. Gerade im 18. Jahrhundert boten sich hier angesichts der Ausdifferenzierung der literarischen Strömungen und Moden zunehmend breitere Möglichkeiten zur Orientierung und ludifikatorischen Zugesellung in ganz verschiedenen Richtungen. Hochschullehrer haben sich an

22 Seine beiden Stammbücher in Göttingen, Niedersächsische Staats- und Universitätsbibliothek: Hist. lit. 48 ha und hb. Einzelne Faksimilia daraus bei Wilhelm Ebel, Göttinger Studenten-Stammbuch aus dem Jahre 1786, Göttingen 1966 (hier Nr. 29).

23 W. W. Schnabel, Das Stammbuch, S. 408-439. 
diesen neuen Spielformen in aller Regel nicht beteiligt und sind stattdessen aus dem Kreis der Albumeinträger ausgeschieden.

$$
* * *
$$

Professoren tauchen freilich nicht nur als Einträger in studentischen (und anderen) Alben auf - sie haben durchaus auch selbst Stammbücher geführt. ${ }^{24}$ Gemeint sind hier nicht die Philotheken, die sie während ihres eigenen Studiums angelegt haben und die nach der Universitätszeit meist abgeschlossen wurden. Im Zentrum stehen sollen vielmehr die (deutlich selteneren) Sammlungen, die sie auch während ihrer Amtszeit fortgeführt oder gar erst angelegt haben. Denn üblicherweise legte man seine Philotheken am Ende des Studiums beiseite und versah sie allenfalls noch mit Hinweisen zum weiteren Lebens- und Karriereweg der eingetragenen Bekannten bzw. mit deren Sterbedaten und einer knappen Segensformel. Wurden sie tatsächlich noch weitergeführt, so ist durchgängig doch immerhin die nachlassende Frequenz der Inskriptionen gegenüber der Studienzeit auffällig; die Alben wurden anderen also nur noch sporadisch zum Eintrag vorgelegt ${ }^{25}$ und noch vor der Berufung auf ein Hochschulamt dann meistens ganz eingestellt. ${ }^{26}$ Deutlich seltener noch sind Belege für die Neuanlage von Stammbüchern, während man bereits ein akademisches Lehramt innehatte. Schließlich war das Führen von Alben doch überwiegend eine Sitte der jüngeren Generation an den Universitäten oder im Kriegsdienst; allenfalls bei Personen mit mobiler Lebensweise (wie etwa Diplomaten, Reisenden oder Exulanten) oder bei Kunsthandwerkern mit einem erlesenen Kundenstamm ${ }^{27}$ lassen sich auch in gesetzterem Lebensalter hin und wieder derartige Alben nachweisen.

Bei Professorenalben sind Beispiele von Mobilität freilich ziemlich selten - schließlich waren die Amtsinhaber beruflich eng an ihren Wirkungsort gebunden und verließen ihn meist kaum noch. Einen Ausnahmefall stellt hier sicher das Stammbuch von Georg Michaelis Cassai (1640-1725) dar. ${ }^{28}$ Der aus Ungarn stammende und an der Universität Wittenberg wirkende Philosophieprofessor - Begründer der dortigen ,Ungarischen

24 Im $R A A$ waren im Februar 2019 insgesamt 326 Stammbücher von (späteren) Professoren recherchierbar. Eine Auswahl derer, die ihre Alben tatsächlich noch in ihrem Hochschulamt geführt haben, reduziert diesen Wert allerdings auf wenige Dutzend.

25 So etwa im Stammbuch des späteren Tübinger Theologieprofessors Johann Friedrich Cotta (1701-1779); Stuttgart, Württembergische Landesbibliothek: Cod. hist. $4^{\circ}$ 55; Digitalisat: < http://digital.wlb-stuttgart.de/sammlungen /sammlungsliste/werksansicht/?no_cache $=1 \&$ tx_dlf\%5Bid $\% 5 \mathrm{D}=6193 \&$ tx_dlf\%5Bpage $\% 5 \mathrm{D}=1>$. Desgleichen das Album des späteren Wittenberger Theologieprofessors Johann Gottlieb Drasdo (1753-1819); dazu Konrad Drasdo, Eintragungen aus dem Stammbuch eines Kursachsen, Archiv für Stamm- und Wappenkunde 13, 1912/13, S. 132 f.; Einträgerverzeichnis auch im RAA.

26 Das Album des späteren Altdorfer Theologieprofessors Jacob Jordan (1556-1632) wurde vor allem während der Studienzeit in den späten 1570er und frühen 80er Jahren geführt (Erlangen, Universitätsbibliothek: Ms. 2444; durchschossenes Exemplar der „Flores hesperidum“ des Christian Egenolf von 1574); Einträge nach seiner Etablierung als Diakon in Altdorf 1585 wurden nur noch gelegentlich getätigt und hingen gelegentlich mit seiner Nebenbeschäftigung als Präzeptor zusammen (S. 34). Sie enden allerdings vor seiner Berufung zum Theologieprofessor 1604. Immerhin führte er bis in die Frühphase seiner akademischen Lehrtätigkeit (bis 1605) in diesem Album einen „Catalogus convictorum meorum“, also eine Auflistung seiner Hausgäste und Kostgänger. Auch diese gab er allerdings mit der Erringung des Lehramts auf.

27 Zum Album eines gefragten Nürnberger Gemmenschneiders Hans Henning, Das Stammbuch von Johann Christoph Dorsch. Blätter der Erinnerung an Gelehrte und Künstler des frühen 18. Jahrhunderts, Marginalien 104, 1986, S. $34-46$.

28 Halle, Universitäts- und Landesbibliothek Sachsen-Anhalt: Ung. Ms. 14. Digitalisat: <http://dlib.bibliothek .uni-halle.de/sammlungen/Stammbuecher/Ung_Ms_14.pdf>. 
Bibliothek' - sammelte darin nicht etwa Einträge seiner Kollegen an der Leucorea; ${ }^{29}$ vielmehr dokumentierte er darin seine rege Reisetätigkeit im mitteldeutschen Raum, die er während seiner letzten beiden Lebensjahrzehnte entfaltete. Auf diesen Reisen führte der Halter, der damals noch als Magister legens und Assessor der Philosophischen Fakultät fungierte, sein Album - das dritte von ihm angelegte - regelmäßig mit. Die Eröffnungsseite adressierte 1707 noch ganz traditionell ,patroni', ,fautores' und , amici', ehe sie in lateinischen Distichen einen kurzen Abriss über das bisherige Leben des Besitzers gab. Dabei vergaß der Verfasser nicht die Huldigung an seine verehrten Vorbilder in der Dichtkunst, unter denen er unter anderem Christian Hoffmann von Hoffmanswaldau (1616-1679) und den jüngeren, ebenfalls in Breslau wirkenden Orientalisten und Theologen Andreas Acoluth (1654-1704) hervorhob. Die Bekanntschaften, die der knapp 70jährige tatsächlich um ein Notat in seinem Album bat, lassen sich dann freilich nur schwer mit dem konventionellen triadischen Schema von Patronen, Gönnern und Freunden fassen. Die Unterscheidung der Gruppen beruhte ja üblicherweise nicht nur auf der unterschiedlichen persönlichen Beziehung, die sie zum Albumhalter unterhielten oder die er sich von ihnen erhoffte; sie beruhte auch auf einer klaren Scheidung von höher- und gleichrangigen Personen, von solchen, von denen er Leistungen erwartete, und solchen, mit denen der Umgang unter Umständen minder berechnend war.

Bei den Einträgern im Stammbuch von Michaelis Cassai handelte es sich häufig um Geistliche und Rektoren städtischer Schulen, aber auch um höhere Beamte in kleinen Orten, ja selbst Handelsleute. In etlichen Fällen wiesen sie sich selbst als ehemalige Schüler des Halters aus, die er in diesen Jahren offenbar zielstrebig besuchte. ${ }^{30}$ Hatte der Wittenberger Hochschullehrer schon in seinen vorangegangenen Alben immer wieder die Autographen aktueller Schüler versammelt, ${ }^{31}$ so scheint das Altersalbum nicht zuletzt von einer nostalgischen Rückschau auf das vergangene Leben geprägt, das dem Lehrbetrieb gewidmet gewesen war; die persönliche Konstellation zwischen dem betagten Sammler und den von $\mathrm{ihm}$ aufgesuchten, deutlich jüngeren Beiträgern scheint dabei erheblich wichtiger gewesen zu sein als die soziale Andienung und Zugesellung, wie sie in den Alben angehender Akademiker oft zu dominieren scheint.

Einen etwas anderen Zuschnitt hatte die Philothek von Nicolaus Rittershausen (1597-1670), die er auch nach seiner Berufung auf eine Professur der Jurisprudenz in Altdorf 1634 weiterführte. ${ }^{32}$ Hier versammelte er überwiegend arrivierte und prominente Besucher in seinem Album, ließ sich aber auch die Chance, außerhalb seiner kleinen Universität Einträge zu akquirieren, nicht entgehen. Dabei kam es ihm entgegen, dass er bei einer im Auftrag des Nürnberger Rates unternommenen Reise zum Regensburger Reichstag 1643 und bei den Friedensexekutionsverhandlungen in der nahen Reichsstadt

29 Einziger Beleg auf fol. 63r: Gottlob Rivinus, Prof. jur., 1711.

30 Etwa fol. 109 ${ }^{\mathrm{r}}, 135^{\mathrm{r}}, 141^{\mathrm{r}}, 165^{\mathrm{r}}, 175^{\mathrm{r}}, 176^{\mathrm{r}}, 18^{\mathrm{r}}$.

31 Das erste Stammbuch (Laufzeit 1671-1711) in Halle, Universitäts- und Landesbibliothek Sachsen-Anhalt: Ung. Ms. 12 (Digitalisat: <http://dlib.bibliothek.uni-halle.de/sammlungen/Stammbuecher/Ung_Ms_12.pdf>), das zweite (Laufzeit 1675-1707) ebd.: Ung. Ms. 13 (Digitalisat: <http://dlib.bibliothek.uni-halle.de/sammlungen /Stammbuecher/Ung_Ms_13.pdf>). Beide Alben haben ihren Schwerpunkt allerdings in den 1770er bis 1790er Jahren.

32 Sein Stammbuch in Nürnberg, Stadtbibliothek: Will III 522 b; dazu Werner Wilhelm Schnabel, Die Stammbücher und Stammbuchfragmente der Stadtbibliothek Nürnberg. 3 Bde. Wiesbaden 1995 (Die Handschriften der Stadtbibliothek Nürnberg, Sonderband), hier Bd. I, Nr. 61 und 63. Die Einträge auch im RAA. 
1649/50 mit allerlei herausgehobenen Juristenkollegen und hochwohlgeborenen Personen in Kontakt kam - eine Chance, die sich ihm in seinem etwas abgelegenen Universitätsstandort so sicher nicht geboten hätte.

Professorenstammbücher gängigen Zuschnitts sind freilich kaum je so ,mobil' gewesen wie in diesen beiden Fällen. Vielmehr wurden sie praktisch ausschließlich am Wirkungsort des Hochschullehrers geführt und haben somit eher den Charakter von Gäste- oder Besucherbüchern. Manchmal wurden sie auch erst dann angelegt, wenn ein Gelehrter einen Wechsel an einen neuen Dienstort vollzogen hatte - sie mochten dann nicht zuletzt durch eine Fremdheitserfahrung motiviert sein, die man ja auch bei den studentischen Alben unterstellen darf, deren Besitzer in aller Regel erstmals ihr Elternhaus verlassen und sich in neue Strukturen einzufinden hatten. ${ }^{33}$

Freilich blieben es eher seltene Sonderfälle, wenn Halter ihr Album in solchen Fällen dazu verwendeten, um darin Inskriptionen der neuen akademischen Kollegen zu sammeln. ${ }^{34}$ Der böhmische Exulant Wilhelm Schwartze/Nigrinus (1588-1638) beispielsweise wurde 1629 Adjunkt der Philosophischen Fakultät in Wittenberg und Anfang des Folgejahres zum Professor der praktischen Philosophie berufen. ${ }^{35}$ Sein Stammbuch hatte er bereits seit seiner Studienzeit 1618 geführt. ${ }^{36}$ Hier nahm er nun 1629-1631 neben diversen Geistlichen tatsächlich einige neue Kollegen aus allen Fakultäten auf, brach diese Praxis aber schnell wieder ab. Die (wenigen) späteren Einträge bis 1636 konzentrierten sich dann auf gelehrte und hochrangige Besucher von außerhalb oder vornehme Studenten, mit denen er zu tun bekam. Offenbar wurde es als unpassend erachtet, wenn ein erwachsener und mit der Berufung auch arrivierter Gelehrter unter Gleichgestellten eine Sammelpraxis fortsetzte, wie sie vor allem von jungen Leuten bekannt war. ${ }^{37}$

Anders sah es dagegen mit Inskriptionen prominenter oder auch weniger prominenter Studenten aus. Diese (und oft auch ihre Präzeptoren) waren bei manchen Professoren, die ihre Philotheken auch noch in fortgeschrittenem Alter führten, durchaus begehrte Einträger. Der Tübinger Theologieprofessor Stephan Gerlach (1546-1612) reaktivierte sein Album,

33 So etwa das Album des Historikers Reiner Reineccius (1541-1595), das auf einem gedruckten Buch (George Buchanan, Paraphrasis Psalmorum Davidis paraphrasis poetica, Antwerpen 1571) basierte und 1582 bei seinem Wechsel von Frankfurt/O. nach Helmstedt angelegt wurde. Kurze Beschreibung: Reiss \& Sohn (Königstein/Ts.), Auktion 159: Wertvolle Bücher, Handschriften. 23.-24. 4. 2013, Nr. 1176; darauf basierende Auflistung einiger Einträger im $R A A$.

34 Gehäuft treten Straßburger Akademieprofessoren etwa im Stammbuch Matthias Berneggers in den Fokus allerdings überwiegend noch in seiner Zeit als Gymnasiallehrer und vor seiner eigenen Berufung an die Akademie und spätere Universität 1613. Eine (unvollständige) Auflistung der Einträger bei Gotthold Lessing, Carl Robert Lessings Bücher- und Handschriftensammlung. Bd. III. Berlin 1916, S. 101-104 bzw. auf dieser Basis im RAA. Grundlage seines Albums war eine Ausgabe der Emblemata des Andrea Alciati, Lyon 1588.

35 Johann Heinrich ZEDLER (Hg.), Großes vollständiges Universal Lexicon Aller Wissenschaften und Künste [...], 68 Bde, Halle - Leipzig 1732-1754, hier Bd. 24, 1740, Sp. 885 f.; Christian Gottlieb Jöcher, Allgemeines Gelehrten-Lexicon [...], 4 Bde, Leipzig 1750 f. (Neudruck Hildesheim 1960 f.), hier Bd. III, 1751, S. 949; Fritz Rотн, Restlose Auswertungen von Leichenpredigten und Personalschriften für genealogische und kulturhistorische Zwecke, VI, Boppard 1970, S. 189 f. (R 5299).

36 Das Album in Oldenburg, Niedersächsisches Staatsarchiv: Best. 297 J, Nr. 49. Die Einträger verzeichnet bei Harald Schieckel, Findbuch zur Stammbuchsammlung 16.-18. Jh. mit biographischen Nachweisen, Oldenburg 1986 (Inventare und kleinere Schriften des Staatsarchivs in Oldenburg, 28), Nr. 49 und darauf basierend im $R A A$.

37 Die gleiche Beobachtung lässt sich auch innerhalb des adeligen Milieus machen, wo die Vorbehalte mitunter dazu führten, dass man die Sammeltätigkeit an junge Familienmitglieder ,delegierte'; vgl. W. W. SchNABEL, Heteronomie. 
das er zuvor in erster Linie während einer Orientreise als Gesandtschaftsprediger geführt hatte, nach der Berufung auf einen Lehrstuhl 1578 zwar gelegentlich, beschränkte sich dann aber darauf, die Notate fürstlicher, adeliger oder besonders prominenter Besucher zu akquirieren. ${ }^{38}$ Auch der Straßburger Rhetorikprofessor Caspar Brülow (1585-1627) versammelte in seinem Album mit Vorliebe adelige und patrizische Studenten. Der Verdacht liegt also nicht ganz ferne, dass das Auswahlprinzip hier eher auf der gesellschaftlichen Geltung der Inskribenten als auf deren akademischen Leistungen beruhte. ${ }^{39}$ In Altdorf legte der Professor der Medizin und Philosophie Ernst Soner (1572-1612) nach seiner Berufung 1605 ein ganz ähnliches Verhalten an den Tag. ${ }^{40}$ Auffällig unter den Inskribenten in seinem Album ist die Häufung polnischer und gelegentlich auch böhmischer Adeliger, die die reichsstädtische Akademie damals häufiger besuchten. ${ }^{41}$ Darunter befinden sich auch mehrere Repräsentanten unitarischer Überzeugungen, die damals für öffentliches Aufsehen sorgten und in ihren Einträgen möglicherweise sogar verdeckte Botschaften hinterließen. In manchen Fällen ist vermutet worden, dass die Stammbucheinträge als verdeckte Kommunikationsmittel im Kreis der Heterodoxen fungiert hätten, die hier Verhaltensempfehlungen oder Anspielungen auf ihre Überzeugung hinterlassen hätten, die nur dem Kundigen im gewünschten Sinne verständlich gewesen seien. ${ }^{42}$ Dass Soner nämlich auch selbst der antitrinitarischen Bewegung anhingt, wurde erst posthum offenbar und führte zu nachdrücklichen Inquisitionen, in deren Folge 1615 auch seine hinterlassenen ,sozinianischen' Schriften öffentlich verbrannt wurden. Soner hat sein Album, in das sich meist ausländische Schüler eintragen durften, bis rund zwei Monate vor seinem Pesttod im Jahr 1612 geführt.

In gleicher Weise für prominente, bereits arrivierte Besucher öffnete später der Altdorfer Professor für Philosophie Johann Albrecht Spies (1704-1766) sein älteres Album, nachdem er 1730 einem Ruf an seine Heimatuniversität gefolgt war; außerhalb seiner Wirkungsstätte und des nahegelegenen Nürnberg akquirierte allerdings auch er keine Einträge mehr. ${ }^{43}$

38 Sein Stammbuch in Stuttgart, Württembergische Landesbibliothek: Cod. hist. $8^{\circ} 120$; dazu Ingeborg KREKLER, Stammbücher bis 1625, Wiesbaden 1999 (Die Handschriften der Württembergischen Landesbibliothek Stuttgart, Sonderreihe, 3), Nr. 15; Auflistung der Einträger auch im RAA.

39 Sein Stammbuch in Göttingen, Niedersächsische Staats- und Universitätsbibliothek: 2 Bibl UFF 553. Basis war ein Bibeldruck von 1607 im damals ungewöhnlichen Folioformat. Dazu Michael Hanstein, Caspar Brülow (1585-1627) und das Straßburger Akademietheater. Lutherische Konfessionalisierung und zeitgenössische Dramatik im akademischen und reichsstädtischen Umfeld, Berlin-Boston 2013 (Frühe Neuzeit, 185), S. 110-120.

40 Sein Stammbuch in Erlangen, Universitätsbibliothek: Ms. 2131. Einträgerverzeichnis im RAA.

41 Theodor Wotschke, Polnische Studenten in Altdorf, Jahrbücher für Kultur und Geschichte der Slaven, NF 4, 1928, S. 216-232; Zdzisław PIetrzyK, Polnische Studenten in Altdorf, in: Hanns Christof Brennecke - Dirk Niefanger - Werner Wilhelm Schnabel (Hgg.), Akademie und Universität Altdorf. Studien zur Hochschulgeschichte Nürnbergs, Wien - Köln - Weimar 2011 (Beihefte zum Archiv für Kulturgeschichte, 69), S. 69-82; Lois EISSNER, Studenten aus Westböhmen an der Hohen Schule zu Altdorf, Der Egerländer 5, 1954, S. 95-98; Heinrich Kunstmann, Die Nürnberger Universität Altdorf und Böhmen. Beiträge zur Erforschung der Ostbeziehungen deutscher Universitäten, Köln-Graz 1963; Martin HoLÝ, Der böhmische Adel und die Akademie in Altdorf, in: H. Ch. Brennecke - D. Niefanger - W. W. Schnabel, Akademie und Universität Altdorf, S. 51-68.

42 Siegfried Frhr. von Scheurl, Die theologische Fakultät Altdorf im Rahmen der werdenden Universität 1575-1623, Nürnberg 1949 (Einzelarbeiten aus der Kirchengeschichte Bayerns, 23), S. 152-154, 156; Cornelia RÉmi, Möglichkeiten heterodoxer Verständigung im Umfeld der Altdorfer Akademie, in: H. Ch. Brennecke D. Niefanger - W. W. Schnabel, Altdorf, S. 167-191, hier S. 180-185.

43 Sein Album in Nürnberg, Stadtbibliothek: Will VIII 123. Dazu W. W. Schnabel, Stammbücher II, Nr. 158 bzw. die Auflistung der Einträger im $R A A$. 
Ein interessantes Beispiel für die Stammbuchführung eines Hochschullehrers bietet die Sammlung von Georg Queck (1561-1628). Bei ihr handelt es sich nicht lediglich um die Fortführung eines älteren Albums unter nun veränderten Bedingungen, sondern um eine Philothek, die erst in Amt und Würden angelegt wurde und von der Forschung bislang nicht zur Kenntnis genommen worden ist. ${ }^{44}$ Queck hatte zunächst als Gymnasiallehrer in Altdorf gewirkt, ehe er 1597 zum Professor der Ethik und der griechischen Sprache an der nürnbergischen Akademie ernannt worden war. Als solcher vertrat er eine traditionelle aristotelische Philosophie und verteidigte die ,Weltweisheit' dabei auch gegen ihre Verächter aus der theologischen Fakultät. ${ }^{45}$ Besonders profiliert scheint seine Lehre nach Ausweis seiner veröffentlichten Disputationen freilich nicht gewesen zu sein. (Abb.6) Während von den früheren Stammbüchern Quecks nur Fragmente auf uns gekommen sind, hat sich sein letztes Album, zwischen 1609 und 1623 geführt, erhalten. Es handelt sich um ein Sammelmedium, das vollständig in seine Amtszeit als Hochschullehrer fällt und dabei auch die eben angedeuteten aufsehenerregenden Auseinandersetzungen um die ,kryptosozinianischen' Umtriebe in Altdorf ${ }^{46}$ und den Übergang von der reichsstädtischen Akademie zur Universität 1622 miterlebte.

Auffällig ist auch bei Queck gerade in der Anfangszeit die Häufung mehr oder minder vornehmer Studenten aus Polen und Böhmen. In nicht weniger als sechs Fällen handelte es sich um Angehörige unitarischer oder anderer heterodoxer Strömungen, die damals bevorzugt gerade Altdorf aufsuchten. ${ }^{47}$ Hier trafen sie auf eine noch relativ tolerante, melanchthonianisch geprägte Atmosphäre, die freilich zunehmend von den Vertretern der lutherischen Orthodoxie eingedämmt und ab 1613 endgültig verdrängt wurde. Auch wenn es sich nicht um eine festgefügte Gruppe von Heterodoxen gehandelt haben wird, erregte die Anwesenheit und das durchaus offene Auftreten profilierter Vertreter des Antitrinitarismus

44 Lemberg, Lwowska Narodowa Naukowa Biblioteka Ukrainy im. Wasyla Stefanyka: Pocz. XVII w. K. 197. Dazu kurz Maciej MATwiJów - Elzbieta OstromecKa, Informator o polonikach w zbiorach rękopismiennych Lwowskiej Narodowej Naukowej Biblioteki Ukrainy im. Wasyla Stefanyka, I, Zbiory Biblioteki Zakladu Narodowego im. Ossolinskich Zinwentaryzowane przed 1945r., Breslau 2010, S. 33, Nr. 806. Weitere Fragmente aus einem Stammbuch Quecks mit meist früheren Einträgen befinden sich in Hannover (Niedersächsisches Landesarchiv: VI Hs. Gr. 13, Nr. 235) und Uppsala (Universitetsbiblioteket: Waller Ms 19).

45 Wolfgang MäHrle, Academia Norica. Wissenschaft und Bildung an der Nürnberger Hohen Schule in Altdorf (1575-1623), Stuttgart 2000 (Contubernium, 54), S. 323-325; Walter Sparn, Aristotelismus in Altdorf. Ein vorläufiges Profil, in: H. Ch. Brennecke - D. Niefanger - W. W. Schnabel (Hgg.), Altdorf, S. 121-150.

46 Gustav Georg Zeltner, Historia Crypto-Socinismi Altorfinae quondam Academiae infesti [...], Leipzig 1729; Scheurl, Die theologische Fakultät Altdorf; Domenico CACCAmo, Sozinianer in Altdorf und Danzig im Zeitalter der Orthodoxie, Zeitschrift für Ostforschung 19, 1970, S. 42-78; Hanns Christof Brennecke, Heterodoxie und sozinianische Häresie in Altdorf, in: H. Ch. Brennecke - D. Niefanger - W. W. Schnabel (Hgg.), Altdorf, S. 151-166. Die kontroverse Forschung zusammenfassend bewertend in jüngerer Zeit Wolfgang MäHrLE, Eine Hochburg des „,Kryptocalvinismus” und des ,, Kryptosozinianismus”? Heterodoxie an der Nürnberger Hochschule in Altdorf um 1600, Mitteilungen des Vereins für Geschichte der Stadt Nürnberg 97, 2010, S. 195-234; Martin SCHMEISSER - Klaus BIRNSTIEL, Gelehrtenkultur und antitrinitarische Häresie an der Nürnberger Akademie zu Altdorf, Daphnis 39, 2010, S. 221-285; Friedrich Vollhardt, Gefährliches Wissen und die Grenzen der Toleranz. Antitrinitarismus in der Gelehrtenkultur des 17. Jahrhunderts, in: Andreas Pietsch - Barbara Stollberg-Rillinger (Hgg.), Konfessionelle Ambiguität als religiöse Praxis in der Frühen Neuzeit, Gütersloh 2013 (Schriften des Vereins für Reformationsgeschichte, 214), S. 221-237.

47 Einschlägig bekannt sind die Inskribenten Stefan von Woynarowa Woynarowski und sein offen unitarischer Informator Michael Güttich/Gittichius im Mai 1609, Adam Sienienski, damals Ehrenrektor der Akademie und Sohn des Besitzers von Rakow, dem Zentrum der polnischen Unitarier, zusammen mit seinem sehr offensiv auftretenden Informator Jan Zaborowski im März 1610, der einschlägig exponierte Martin Ruarus im Juni 1611 sowie Christoph Sieniuta im April 1614. 
bzw. die Sympathie intellektuell brillanter Hochschullehrer doch eine Aufmerksamkeit, die sich zu einem offenen Konflikt auswuchs. ${ }^{48}$

Nun ist von heterodoxen Neigungen des Altdorfer Ethikers und Gräzisten nichts bekannt - in den Kreis der Verdächtigen, gegen die erst 1615-1617 nachhaltiger ermittelt wurde, ist Queck offenbar selbst nicht geraten. ${ }^{49}$ Seine Stellung ist allerdings dadurch interessant, dass er als Rektor auf der einen Seite 1616 mit der Inquisition der Heterodoxen in Altdorf betraut wurde, ${ }^{50}$ auf der anderen aber über mehrere Jahre von deren Anwesenheit profitiert hatte. Denn in ihren Einträgen offenbarten sich die Studenten und Reisenden aus den osteuropäischen Ländern nicht selten als ,hospites', waren also Hausgäste bzw. Kostgänger Quecks. Wie viele andere verdiente sich der Altdorfer Professor, der nur schlecht besoldet und dessen griechisches Lehrangebot nur wenig angenommen wurde, durch die Aufnahme von Fremden ein Zubrot. Etliche von ihnen werden - auch wenn das nur vereinzelt signalisiert ist $-\mathrm{zu}$ den Zahlern seiner Privatlektionen gehört haben. $\mathrm{Zu}$ ihnen bestand also durchaus ein engeres persönliches Verhältnis, das über die rein institutionelle Lehrer-Schüler-Beziehung hinausging. Beim Abschied von der Altdorfer Hochschule dokumentierten die Kostgänger denn auch gerne ihre Verbundenheit in dem Stammbuch, das ihnen Queck vorlegte. In einem lateinischen oder gar griechischen, oft bemüht akkurat geschriebenen Eintrag gingen sie nicht selten auf die staatsethischen und politikwissenschaftlichen Interessen des Albumhalters ein. Zudem haben die vornehmeren unter ihnen immer wieder auch gouachierte Wappen hinterlassen, die von spezialisierten Briefmalern angefertigt wurden.

Zugleich bemühten sich die Einträger offenbar gezielt darum, dem Altdorfer Professor Belege ihrer Wertschätzung und Neigung zu hinterlassen. Lediglich zwei adelige Inskribenten verzichteten auf eine ausführliche Zueignungsformel und versahen die eingetragene Sentenz lediglich mit ihrer Unterschrift. Die übrigen - adelige wie patrizische und bürgerliche - sparten hingegen nicht mit Formeln der Ehrerbietung gegenüber dem akademischen Lehrer. Die rühmenden Auszeichnungen sind dort bezeichnenderweise sehr viel ausführlicher als bei Professoreneinträgen ihrerseits. Sie mochten dem Empfänger im abgelegenen Altdorf das Bewusstsein verschaffen, weit über die engen Grenzen des nürnbergischen Territoriums Bekanntheit und Achtung zu genießen.

Fast durchgängig konnte sich der Adressat so mit dem Epitheton ,clarissimus' bedacht sehen; nicht selten wurde es mit Adjektiven wie ,excellentissimus', ,praestantissimus', ,dignissimus' oder ,magnificus' ergänzt - superlativische Formeln, die freilich eher Höflichkeitsfloskeln ohne genaueren Beschreibungswert waren. In einzelnen Fällen wurde der Lobpreis allerdings auch durchaus genauer spezifiziert: fachlich konnte sich Queck dort als ,doctissimus' und ,cum doctrina tum literatura et scientiis clarissimus' (194) gewürdigt sehen; gerne wurde auch seine moralische Tugendhaftigkeit mit Formeln wie ,antiqua fide et illibata probitate splendissimus' (148) oder als ,omnibus virtutibus praeclarus' (25) gerühmt. Mit solchen Belegen (die von einem missgünstigen späteren Besitzer übrigens gelegentlich auch gestrichen wurden) wurden dem Albumhalter zwar Komplimente

48 W. MÄHRLE, Eine Hochburg.

49 Bezeichnenderweise ergibt sich auch nur in einem einzigen Fall - dem des Michael Güttich - eine Überschneidung mit den einschlägig bekannten Antitrinitariern aus dem Album Soners.

50 Karl Braun, Der Socinianismus in Altdorf 1616, Zeitschrift für bayerische Kirchengeschichte 8, 1933, S. $65-81,129-150$, hier S. 80. 
zuteil - einer Öffentlichkeit außerhalb des Kreises der meist noch jungen Besucher und Kostgänger hat er sie aber wohl kaum zugänglich gemacht. Eine mehr oder minder öffentlichkeitswirksame ,Zugesellung' zu einem bestimmten Milieu und zu höheren Statusgruppen, wie das bei studentischen Stammbüchern der Zeit oft augenfällig ist, war damit also kaum verbunden.

Dafür war der Kreis der Inskribenten wohl auch zu inhomogen. Innerhalb des akademischen Milieus standen die Einträger fast sämtlich auf einer niedrigeren Statusstufe; sie fühlten sich als Studenten dem Lehrer und Quartiergeber verpflichtet. Sozial hingegen genoss zumindest ein Teil von ihnen einen superioren Rang, da ihnen als Adeligen oder Patriziern eine höhere gesellschaftliche Reputation zukam als dem Professor. Während studentische Alben in aller Regel Einträge von Personen mit höherem oder zumindest gleichem, nur selten aber von niedrigerem Status versammelten, führte der Gästebuch-Charakter des Professorenalbums zu einer anderen sozialen Mischung der Inskribentenschaft. Diese war im allgemeinen auch untereinander nicht näher vernetzt, sondern fokussierte sich im Stammbuch ausschließlich auf ihre Relation zum Gastgeber. Eine Verbindung innerhalb des Gelehrtenmilieus markieren einzig zwei Inskriptionen: ein längerer griechischer Eintrag des reisenden Bischofs Metrophanes Kritopoulos (98), von dem Queck auch eine griechische Rede ins Lateinische übersetzt und ediert hatte, und ein solcher des Berliner Arztes Georg Nößler (108), der Queck 1617 aufsuchte und später selbst Professor der Medizin in Altdorf werden sollte.

Auch Quecks Professorenstammbuch diente demzufolge nicht mehr primär dem Wunsch nach nützlichen Kontakten und als Dokument des Vernetzungswillens, der Integration in einem Beziehungssystem und der persönlichen Positionierung, wie es bei seinen eigenen früheren Alben noch naheliegt; es fungierte tatsächlich wohl eher als persönlicher Erinnerungsanreiz an Mieter und Gäste, die er in seinem Haus beherbergt hatte. Eine solche Dokumentation von Haus- und Tischgemeinschaften lässt sich im universitären Milieu auch in anderer Form immer wieder beobachten: Nicolaus Taurellus (1547-1606) hat sie um 1600 - ebenfalls in Altdorf - für eine Frühform des Subskriptionsverfahrens genutzt und die Picturae seiner Emblematik von seinen Tischgästen finanzieren lassen; ${ }^{51}$ von studentischer Seite wurde sie in einer Fülle von Gelegenheitsdrucken zu freudigen und traurigen Anlässen dichterisch umgesetzt.

Der Wunsch, materielle Erinnerungen an Studenten zu bewahren, die im Idealfall von höherem Stande waren und unter den Bedingungen einer ständisch geschichteten Gesellschaft wie der frühmodernen auch ein wenig zur Hebung der eigenen Reputation beitrugen, zeigt bei den Professorenalben zwar durchaus Parallelen zu den Stammbüchern zeitgenössischer Studenten - ihr Einträgerkreis, die Eintragssituationen und ihre Ortsstabilität belegen aber zugleich einen ganz anderen Zuschnitt, der sie als eigenständige Subtypen der Alben aus dem Gelehrtenmilieu ausweist.

$* * *$

Stammbücher ermöglichen auf diese Weise interessante Einblicke in die kulturellen Praktiken des Professorenstandes, freilich weniger in das Alltagsleben der Betreffenden

51 Werner Wilhelm Schnabel, Über das Dedizieren von Emblemen. Binnenzueignungen in Emblematiken des 16. und 17. Jahrhunderts, in: F. van Ingen - Ch. Juranek, Ars et Amicitia, S. 115-166, hier S. 151-157. 
als vielmehr in deren Strategien der Selbstdarstellung, Positionierung und Relationierung gegenüber anderen Ständen und Milieus. Gesellschaftliche Komments - wie die Selbsteinordnung im hierarchischen Gefüge der sozialen Stände ${ }^{52}$ und universitären Fakultäten ${ }^{53}$ wurden von den erfahrenen Schreibern durchgängig eingehalten. Und auch die gewählten Einträge waren in aller Regel keine, gedankenlosen' Floskeln, sondern bewusst gewählte Sentenzen oder Zitate, die direkt oder indirekt Informationen über den Schreiber, dessen Ausrichtung und Selbstbild vermitteln sollten. Nicht nur innerhalb des akademischen Kosmos, sondern ebenso gegenüber Außenstehenden galt es mithilfe der Themen, Inhalte und Referenzen, aber auch durch die aufgezählten Statusangaben die eigene Würde und Reputation zu beweisen. Sie sollten auch für spätere Leser festgehalten werden.

Dazu gehörte durchgängig ein belehrender, konstatierender, mahnender, auffordernder Redegestus, der überlegene, axiomatische Wahrheiten verkündete oder auch reflektierte, der Ernsthaftigkeit, moralische Festigung und Sittenstrenge des Schreibers, aber auch seine Vertrautheit mit den Weltläuften und den Schwierigkeiten der Selbstbehauptung durchscheinen ließ. Einsicht in die, großen' Dinge wurde offensichtlich erwartet und dementsprechend auch markiert. Fakultativ konnten dazu noch Signale fachlicher Überlegenheit treten, die der Einträger durch die Wahl der Sprache, eines Inhalts oder einer Zitatreferenz an den Tag legte und unter Umständen mit kommentierenden Bemerkungen unterstrich. Derartige Verfahren der Selbstdarstellung lassen sich praktisch durchgängig beobachten. Verzichtet wurde dagegen in aller Regel auf pikturale Beigaben: Wappen oder emblematische Darstellungen blieben ein Reservat des Adels und Patriziats bzw. der bildungsbeflissenen Akademikerschaft außerhalb des Professorenstandes, Noten ein solches musikbegeisterter Kommilitonen oder von Berufsmusikern. Mit der nahezu durchgängigen Beschränkung auf Textbeiträge in gelegentlich recht nachlässiger Schrift signalisierten die Hochschullehrer Ernsthaftigkeit, aber auch eine gewisse ,Trockenheit' oder ,Sprödigkeit', die durchaus mit zum Stereotyp ihres Standes gehörte.

Auffälliger ist ein Veränderungsprozess, der auf der Oberflächenebene zunächst einmal rein quantitativ erscheint: In der Frühzeit waren die schriftlichen Beiträge meist noch vergleichsweise ausführlich; später gerieten sie oft ausgesprochen knapp. Dieser längerfristige Wandel hat freilich auch eine qualitative Bedeutung, die über das Abzählen von Zeilen oder Wörtern hinausreicht: mit ihm wurde im Laufe des 17. und vor allem 18. Jahrhunderts zugleich eine zunehmende Distanz zwischen Schreiber und Empfänger aufgebaut. Mögen sich beide Akteure zuvor als gemeinsame Vertreter einer elitären Bildungsschicht auf humanistischer Grundlage verstanden haben, so wurde der Abstand zwischen den Lehrenden auf der einen und den Lernenden auf der anderen Seite auch in den Albumeinträgen immer deutlicher. Dies beruhte nicht etwa auf der Entwicklung zu einer ,Massenuniversität',

52 Professoreneinträge erscheinen in der Regel nach denen des Fürstenstandes, des Adels und des höheren Kirchenpersonals, aber vor denen niederrangiger Kirchenleute, Rektoren und Lehrer. Studentische Inskriptionen beginnen meist erst in der zweiten Hälfte der Alben.

53 Auch im Stammbuch wurde die Hierarchie von Theologen, Juristen, Medizinern und Philosophen meist eingehalten und allenfalls dort außer Kraft gesetzt, wo ein Rektorenamt eine besondere Stellung markierte. Allerdings gab es zu allen Zeiten Beispiele des ,Gelehrtenstolzes' und der Konkurrenz, die sich mitunter auch in der Relationierung innerhalb der Alben niederschlugen. Zu möglichen Organisationsschemata W. W. ScHNABEL, Das Stammbuch, S. 138-141. 
nahmen die Immatrikulationszahlen im 18. Jahrhundert doch nahezu permanent ab. ${ }^{54}$ Die mentale Entfernung scheint eher ein sozialpsychologisches oder auch soziologisches Phänomen gewesen zu sein. Sie hatte möglicherweise auch mit einer Pluralisierung milieuspezifischer Werthorizonte und einer deutlichen Auseinanderentwicklung professoraler und studentischer Kulturen zu tun.

Studentische Alben mit professoralen Einträgen sind dabei nicht nur Dokumente eines solchen Prozesses, sondern auch selbst dessen Opfer geworden. Denn die Distanzhaltung hatte durchaus auch Auswirkungen auf Reputation und Stellenwert der Stammbücher an sich: Mit dem zunehmenden Verzicht auf ,persönliche', zugewandte Formulierungen, aber auch dem Tilgen eigener Statusangaben wurde nicht nur der ,Schreibaufwand' des höherrangigen Inskribenten verringert, sondern verloren die Einträge auch die ausgestellte Akzentuierung als Dokumente zwischenmenschlicher Beziehung. Ohne Zweifel förderte eine solche Praxis eine Wahrnehmungsweise, derartige Notate nur noch als Zeugnisse einer individuellen Handschrift oder als bestenfalls , auratisches' Relikt einer bekannten Persönlichkeit zu betrachten, nicht mehr als persönlichen Niederschlag einer befruchtenden Begegnung. Damit wurden die Einträge zugleich einer Beliebigkeit verdächtig, die ihren Wert als ,symbolisches Kapital' schmälerte und damit auch die Wertigkeit des Sammelmediums beeinträchtigte. Das Misstrauen gegenüber der moralischen ,Wahrhaftigkeit' von Albuminskriptionen war also nicht der einzige Faktor, der eine Bedeutungsverschiebung der Stammbuchkultur einläutete; 55 er mag auch mit der Verweigerung unmittelbarer Zugewandtheit zu tun gehabt haben, wie sie im Verkehr zwischen den beiden Statusgruppen mehr und mehr sichtbar wurde. In einem zeitgenössischen Kontext, der dem Freundschaftskult einen besonderen, sentimentalen Stellenwert zuwies, wurde so erst recht der Eindruck von Differenz verstärkt, während die identitäre Bindung an die eigene Gruppe zunahm. Wenn die Einträge des universitären Lehrpersonals über kurz oder lang aus den studentischen Alben verschwanden, so zeigt dies klar, dass die Stammbücher eben nicht nur ein Medium der Erinnerungspflege oder Memorialkultur waren, sondern zugleich der Zuordnung zu oder Abgrenzung von Gruppen dienten, die soziale Identität generierten. ${ }^{56}$

Wesentlich schwieriger erscheinen die Verhältnissen bei den Professorenalben - und das nicht nur, weil die Zahl untersuchbarer Exemplare weitaus kleiner ist. Professorale Stammbücher müssen insgesamt als Ausnahmeerscheinung angesehen werden, die angesichts ihrer variablen Verwendungsverhältnisse oft eine sehr viel individuellere Prägung erfuhren als die Sammelmedien der Studentenschaft. Insgesamt weisen sie nur eine vergleichsweise geringe Zahl von Einträgen auf und wurden nur über einen recht begrenzten Zeitraum geführt. Mehrheitlich waren es dann die frühen Berufsjahre, in denen ein bereits existierendes, aus der eigenen Studentenzeit stammendes Album sporadisch mit weiteren Einträgen gefüllt wurde. Innerhalb weniger Jahre ließ das Interesse an der Sammelform dann aber in der Regel auch wieder nach und führte zur Einstellung der Akquisitionstätigkeit. Die

54 Franz Eulenburg, Die Frequenz der deutschen Universitäten von ihrer Gründung bis zur Gegenwart, Leipzig 1904 (Abhandlungen der Philologisch-Historischen Klasse der Königl. Sächsischen Gesellschaft der Wissenschaften, 24/II). Graphische Umsetzung bei Ulrich RAsche, Immatrikulationen in Altdorf 1581-1810, in: Werner Wilhelm Schnabel (Hg.), Athena Norica. Bilder und Daten zur Geschichte der Universität Altdorf, Nürnberg 2012 (gff digital, A 3), B9100.

55 W. W. SCHNABEL, Stammbuch-Schelte.

56 Neuerdings Werner Wilhelm Schnabel, Der Philothecarius à la mode. Stammbuchpraxis als Projektionsraum studentischer Gruppenkulturen, Archiv für Kulturgeschichte 102, 2020, S. 87-132. 
Zahl der Belege ist also durchaus nicht so groß, dass sie eindeutige und breit unterbaubare Aussagen zuließe.

Immerhin scheint man festhalten zu können, dass die soziale Ausrichtung in Professorenstammbüchern eine andere war als in denen von Studenten. Kann bei diesen meist eine Orientierung ,nach oben' (Gönner, Förderer, Professoren und Honoratioren) oder , auf gleicher Ebene' (Kommilitonen), kaum je dagegen eine ,nach unten' (Wirte, Kaufleute, Handwerker) beobachtet werden, so sind die Professorenstammbücher mit solch einfachen sozialen Konturierungen und Statusrelationen kaum in den Griff zu bekommen. Ausnahmen scheinen lediglich die Alben gewesen zu sein, in denen ein Halter Inskriptionen , auf gleicher Ebene' gesammelt hat. Professorenalben mit Professoreneinträgen sind demzufolge außerordentlich selten. Allenfalls waren es durchreisende Honoratioren oder außergewöhnliche Besucher, die um ein Notat gebeten wurden; meist gehörten sie auch nicht etwa der eigenen Disziplin an, sondern rekrutierten sich aus herausgehobenen Vertretern anderer Fachgebiete (also etwa Superintendenten, Vertreter anderer Konfessionsgruppen, Diplomaten etc.). Hier scheint also nicht die ,Zugesellung' zu einer Gruppe, sondern eher das Festhalten einer außergewöhnlichen Situation oder Begegnung eine ausschlaggebende Motivation gewesen zu sein. Von Ausnahmefällen abgesehen, blieben auch höchstrangige Kontakte unberücksichtigt: Inskriptionen von Fürsten und (erwachsenen) Hochadeligen findet man vor allem in adeligen Alben, im akademischen Milieu hingegen eher in studentischen als in professoralen Stammbüchern. Auch der ,Andienungs'-Aspekt scheint bei den Hochschullehrern also keine sonderliche Bedeutung gehabt zu haben.

Dagegen konnten sich in den Sammlungen der Professoren recht häufig Studenten verewigen. Dabei handelte es sich meist nicht etwa um ,Meisterschüler', denen später eine glanzvolle Karriere zugetraut wurde und die sie dann auch gemacht haben. Vielmehr scheinen die Halter vor allem ihre Hausgäste um einen Eintrag gebeten zu haben. Wenn diese gar einen Adelstitel vorweisen konnten oder von fernher kamen und damit über einen gewissen ,Exotismus'-Charakter verfügten, war dies sicher durchaus erwünscht, weil es den Attraktionswert des eigenen Haushalts und nicht zuletzt den der eigenen Person dokumentierte - Bedingung war es allerdings nicht. Es scheint eher der direkte Kontakt, das Zusammenleben mit den jungen (zahlenden) Gästen über einen gewissen Zeitraum gewesen zu sein, was die älteren Stammbuchführer zu ihrer Sammeltätigkeit bewog. Um ein Notat gebeten wurde, wer im Hause lebte (bzw. sich nach seinem Aufenthalt verabschiedete). Akquisitionsbesuche oder gar Reisen zu auswärtigen Berühmtheiten spielten dagegen in aller Regel keine Rolle. Die Alben blieben also ganz überwiegend ortsstabil oder machten nur in nächster Umgebung die Runde. Insbesondere scheinen hinter der professoralen Sammeltätigkeit keine ausgeprägt strategischen Überlegungen gestanden haben, wie sie bei den Studierenden nicht selten waren. ${ }^{57}$ Auch ein deutlicher Vernetzungswillen lässt sich nicht erkennen, fehlen doch meist auch Anmerkungen, die über die spätere Schicksale der Einträger berichten. Der weitere Lebensweg der Einträger geriet also offenbar aus dem Fokus, dauernde Kontakte scheinen sich nicht entwickelt zu haben oder galten nicht als dokumentationswürdig.

Seitens der studentischen Inskribenten stellten die Einträge in professorale Alben immerhin eine gewisse Herausforderung dar. Adelige Schreiber ließen hier zwar mitunter

57 Vgl. etwa W. W. Schnabel, Johann Wilhelm Bergius. 
deutlich erkennen, dass sie sich ihrer geburtsständischen Superiorität sehr wohl bewusst waren - Wappenillustrationen mit sehr knappen Texteinträgen und gelegentlich nur mit einer Unterschrift ohne Dedikationsformel markierten den sozialen Abstand auch gegenüber dem überwiegend bürgerlichen Lehrpersonal. Studenten minderer Herkunft konnten sich solche Praktiken dagegen nicht erlauben. Sie versuchten sich ihrerseits als ernsthafte und strebsame Schüler zu verewigen, die auch in Thema und Eintragsgestaltung ganz den erwarteten Rollenvorstellungen entsprachen. Es sind deshalb neben biblischen Sprüchen v.a. philosophische Einsichten in den Lauf der Welt oder zielgerichtete Appelle an sich selbst, die hier festgehalten wurden. Dabei war es sicher ratsam, auf die Interessengebiete und Überzeugungen des Hausherrn entsprechend einzugehen. Besonderer Stellenwert kam daneben auch dem Lob des Professors zu, an dessen Tisch man verkehrte. Er wurde im Zueignungsteil nicht nur mit der Benennung des persönlichen Verhältnisses, sondern auch mit rühmenden Adjektiven passend platziert. Einträge dieser Art waren sicher keine genuinen Ehrfurchts- oder Neigungsbekundungen, sondern ein Ritual, bei dem man mit konventionalisierten sprachlichen Mitteln seine Gewandtheit zu zeigen und seine Dankbarkeit zu bezeugen hatte.

So zeigen sich die Professorenalben als eine Sondergruppe innerhalb des Stammbuch-Kosmos, der in seinen diachronen und synchronen, sozialen bzw. milieuspezifischen Ausdifferenzierungen vom 16. Jahrhundert bis heute keineswegs hinreichend erforscht ist. Dass die zunehmende Digitalisierung der Unikate, die Möglichkeit zum Auffinden entsprechender Spezimina und das steigende Interesse weit über die wissenschaftliche Öffentlichkeit hinaus verstärkte Anreize für die Beschäftigung mit dieser vielseitig nutzbaren Handschriftengattung bietet, ist überaus erfreulich. Aufmerksamkeit hat aber auch den Rollen und Verhaltensweisen der Akteure zu gelten, die durchaus unterschiedlich sein können - je nachdem, ob sie als Inskribenten oder als Albumhalter fungieren. Stammbücher wie Einträge zeigen sich dabei als Medien eines Kommunikationsprozesses, der von der jeweilig aktuellen Situation und den Personenkonstellationen geprägt ist, mit der Einbeziehung künftiger Leser und deren kalkulierten Wahrnehmungsweisen und Reaktionen aber letztendlich weit darüber hinausreicht. Diese wirkungsbezogenen intentionalen Aspekte ernst zu nehmen und nicht nur holzschnittartig, sondern differenziert zu bestimmen, ist eine Aufgabe, die noch zu lösen sein wird. Neben den dazu nötigen Einzelfalluntersuchungen sollte sie immer auch den Versuch von Typenbildungen beinhalten, der es im Abgleich mit anderen erst erlaubt, den Stellenwert oder die Repräsentativität der individuellen Relikte zu bewerten.

WERNER WILHELM SCHNABEL

\title{
PROFESOŘI V PAMÁTNÍCÍCH - PAMÁTNÍKY PROFESORŮ. KONSTELACE ROLÍ A INSCENAČNÍ PRAXE
}

\author{
RESUMÉ
}

Studie sleduje univerzitní profesory jako původce (majitele) a inskribenty v památníkové kultuře raného novověku. Obsahuje analýzu profesorských památníků (štambuchy, alba amicorum) jako jednoho ze specifických typů tohoto pramene. $\mathrm{V}$ českém prostředí nejsou profesorské památníky v klasické podobě zastoupeny, proto si 
jich česká literatura dosud málo všímala. Článek vychází z databáze Repertorium alborum amicorum (RAA). První část studie naznačuje komunikační zvyklosti spojené s fenoménem raně novověkých památníků. Druhá se zaměřuje na profesory coby inskribenty do památníkủ. Pozornost věnuje charakteru zápisů. Evidentní je častá strohost jejich zápisů. Mezi specifika profesorských záznamů ve studentských památnících patří i užití různých jazyků, zejména latiny a řečtiny (i v době, kdy již měla převahu němčina) jako demonstrace ,učenosti“. Třetí, nejrozsáhlejší část studie je věnována profesorům coby původcům (vlastníkům) památníků. Mezi záznamy jsou připomenuta i bohemika, ale rozsáhlejší prostor se dostává jen památníkovému odkazu Georga Quecka (15611628), působícího v Altdorfu. Pozornost se obrací i na formu věnování nebo záznamy prominentních studentů v profesorských památnících.

Památníky představují osobitou formu sociální sebereprezentace úzce spjatou s akademickým a učeneckým prostředím. Byly to komunikační prostředky, jejichž podoba byla ovlivněna konkrétní situací jejich majitelů, vztahem mezi nimi a zapisovateli i jejich společenskou rolí.

(český překlad Blanka Zilynská)

Prof. Dr. Werner Wilhelm Schnabel

Department Germanistik und Komparatistik

Friedrich-Alexander-Universität Erlangen-Nürnberg

werner.w.schnabel@fau.de

\section{Abbildungen:}

Die erste Abbildung s. die Farbanlage, S. 279.

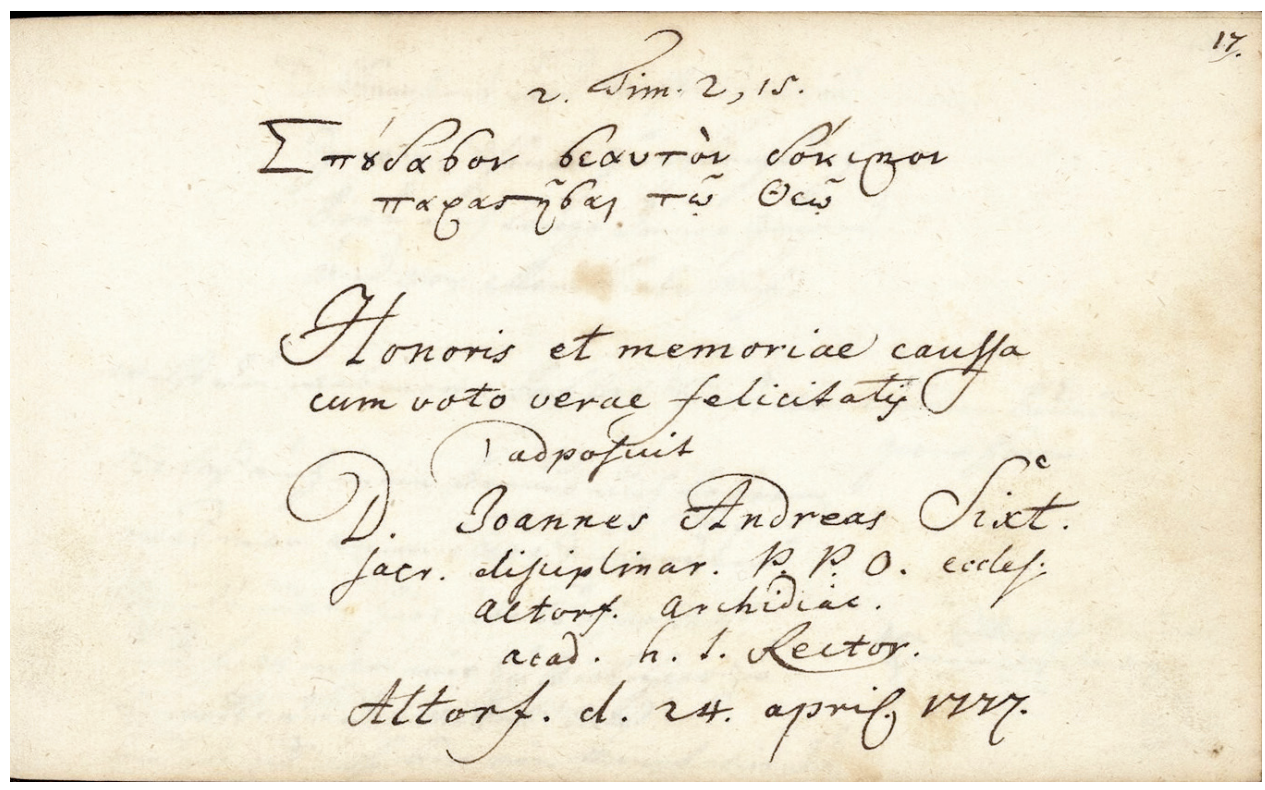

Abb. 2: Eintrag Eintrag Johann Andreas Sixt (1742-1810), Altdorf 1777, im Stammbuch Johann Friedrich Seiferheld ( $\left.{ }^{*} 1757\right)$. Straßburg, BNU: Ms. 2.143, fol. $17^{r}$. 


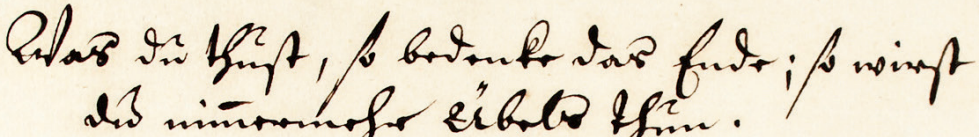

Dex.d. IIICHä̈MDCCLXVI.
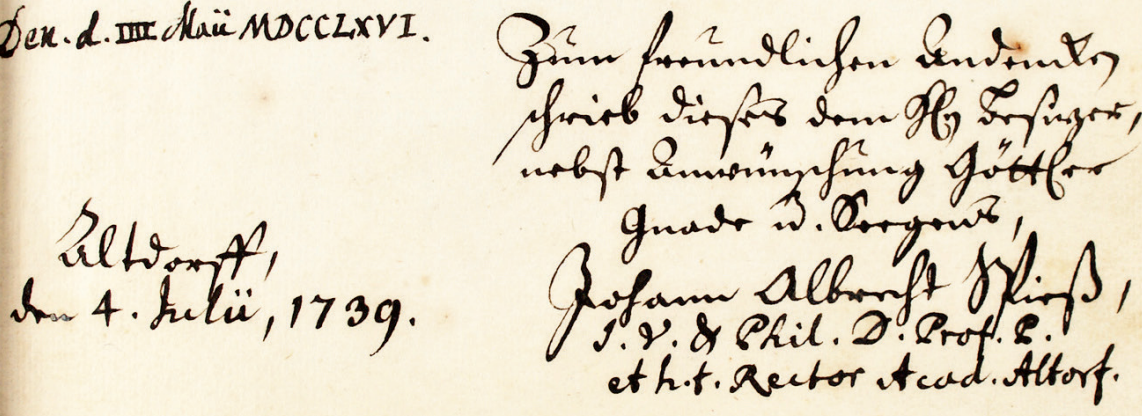

Abb. 3: Eintrag Johann Albrecht Spieß (1704-1766), Altdorf 1739, im Stammbuch Lorenz Schüpfel (1716-1789). Erlangen, UB: Ms. 1371, fol. 39r.

Ton unimi parum cognosfe, fed in parum cognito ficte et dui porfererasfe turpel est.

Berolini d. 28 Stlast.

$$
\begin{aligned}
& 1747
\end{aligned}
$$

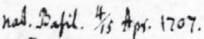

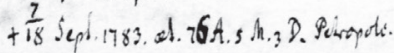


adore dieus fois juste et iheri In patrie: volt.

them ethon.e: for.

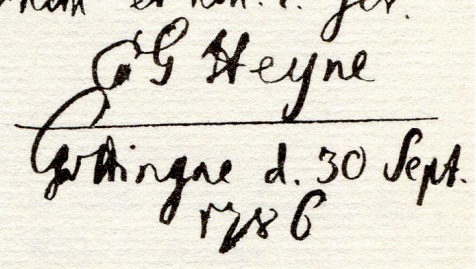

Abb. 5: Eintrag Christian Gottlieb Heyne (1729-1812), Göttingen 1786, im Stammbuch Sándor von Podmaniczki. Göttingen, NsSUB: Hist. lit. 48 ha.

123 


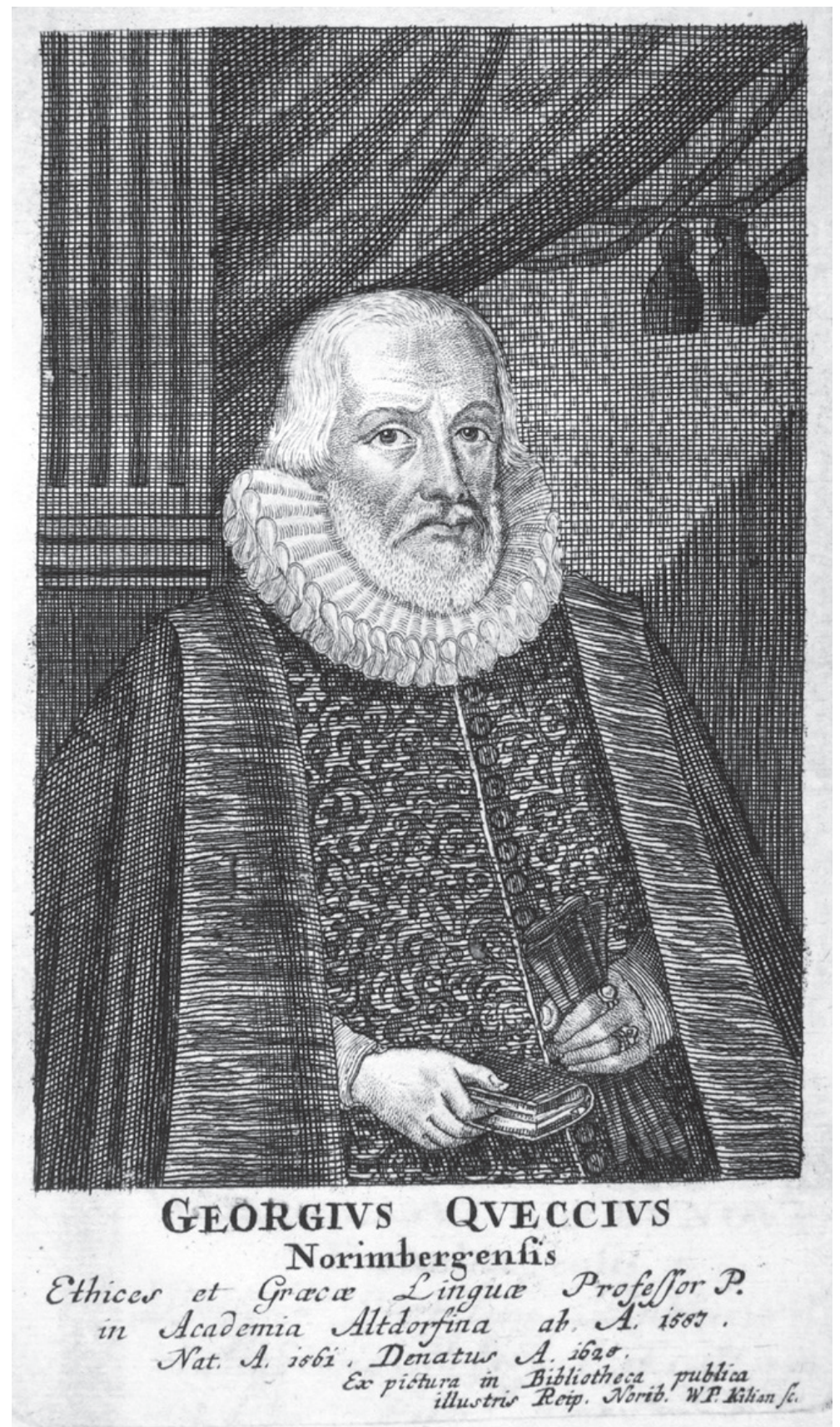

Abb. 6: Georg Queck (1561-1628). Kupferstich von Wolfgang Philipp Kilian (1654-1732) in: Friedrich Rотн-Scholtz (Hg.), Icones Ervditorvm Academiae Altdorfinae [...], Nürnberg-Altdorf 1721/23, Nr. [98]. 